\title{
Article \\ A Study on a New Method for Flexoelectric Coefficient Estimation of the Flexoelectric Unimorph Sensing Element
}

\author{
Seol Ryung Kwon ${ }^{1, *}$ and Yongrae Roh ${ }^{2}$ (D) \\ 1 Korea Institute for Robot Industry Advancement, Daegu 41496, Korea \\ 2 School of Mechanical Engineering, Kyungpook National University, Daegu 41566, Korea; yryong@knu.ac.kr \\ * Correspondence: seolkwon@kiria.org; Tel.: +82-10-8584-3646
}

check for

updates

Citation: Kwon, S.R.; Roh, Y. A Study on a New Method for Flexoelectric Coefficient Estimation of the Flexoelectric Unimorph Sensing Element. Appl. Mech. 2021, 2, 841-848. https://doi.org/10.3390/ applmech 2040048

Received: 23 September 2021

Accepted: 12 October 2021

Published: 15 October 2021

Publisher's Note: MDPI stays neutral with regard to jurisdictional claims in published maps and institutional affiliations.

Copyright: (c) 2021 by the authors. Licensee MDPI, Basel, Switzerland. This article is an open access article distributed under the terms and conditions of the Creative Commons Attribution (CC BY) license (https:/ / creativecommons.org/licenses/by/ $4.0 /)$.

\begin{abstract}
In a flexoelectric sensing element using bending mode, estimation of the flexoelectric coefficient was investigated using 3-D stress/strain analysis and experiments. The proposed method uses the results (deformation and strain) from the finite element analysis (FEA). The estimated flexoelectric coefficients were compared with those obtained via the conventional method (Euler's beam theory) under the assumption of the quasi 1-D stress field. The results show that the RMS value and standard deviation of the estimated flexoelectric coefficient for the 3-D stress field case of the sensing element are $31.51 \mu \mathrm{C} / \mathrm{m}$ and $0.24 \%$, respectively. In addition, we found that the flexoelectric coefficient obtained from the results of the 3-D stress analysis is $1.8 \%$ smaller than that of the quasi-1-D stress analysis. Therefore, in order to obtain a more reliable flexoelectric coefficient in the sensing element, the results of the 3-D numerical stress analysis should be used for accurate estimation of the flexoelectric coefficient.
\end{abstract}

Keywords: bending mode; strain gradient; stress analysis; flexoelectric coefficient; flexoelectricity; unimorph sensing element

\section{Introduction}

To meet the strict requirements of the rapidly developing industry in different fields, a sensing mechanism using the flexoelectric effect was first introduced and researched by Kogan [1], as an alternative to the sensing mechanism using the piezoelectric effect. The piezoelectric effect produces electric charge according to the induced stress/strain generated by mechanical inputs such as force or pressure. By contrast, the flexoelectric effect generates the electric charge according to the induced strain gradient.

The flexoelectric effect has several unique properties over other sensing mechanisms, such as the lack of aging problems and broad material choice. In particular, the scaling effect is the most outstanding feature of this sensing mechanism. This effect enables sensors to be micro/nano miniaturized, as the charge output is generated based on the strain gradient $[2,3]$. In general, there are three modes in which the flexoelectric effect can be exploited, namely, longitudinal, shear and bending modes [4]. Among these, the structure of the longitudinal and shear modes is quite robust, so these modes are more suitable for sensing high-strength incident signals. However, since the induced strain gradient is small, the charge output generated at the sensing element is also small.

On the other hand, structures in the bending mode can induce a high strain gradient, leading to a large flexoelectric output and high sensitivity [5]. In this respect, the bending mode is typically used to design several types of sensors where the amplitude of the incident signal is infinitesimal. As an example, the flexoelectric accelerometer and microphone $[5,6]$ use the bending mode for their sensing ability. In addition, Huang et al. [7] investigated a flexoelectric curvature sensor using the bending mode by attaching the sensor on the side surface of a target structure.

It is well known that the accuracy of a sensor depends on the linearity between the input signal strength and electric output. Until recently, for the sake of simplicity, the 
flexoelectricity of a sensing element was calculated using the analytical results of the stress and strain obtained under the assumption of the quasi 1-D stress field for the sensing element [8].

The following equation describes the flexoelectric effect.

$$
P_{l}=\mu_{i j k l} \frac{\partial \varepsilon_{i j}}{\partial x_{k}}
$$

where $P_{l}$ is the flexoelectric polarization, $\mu_{i j k l}$ is the polar tensor flexoelectric coefficient, $\varepsilon_{i j}$ is the elastic strain and $x_{k}$ is the position coordinate.

As it can be deduced from Equation (1), the charge output is linearly proportional to the input when it is under a 1-D stress field. However, the stress field in the sensing element is a 3-D problem in practical cases. Therefore, it is natural that the actual distributions of stress and strain in the sensing element are quite different from those of the quasi-1-D analytical analysis, which can result in the discrepancy between the incident signal strength and measured charge output [9].

In these connections, for the sensing element of the unimorph mode, the present work proposes a new method for estimating the flexoelectric output and flexoelectric coefficient, which consists of using the results of the 3-D structural analysis and experiments with a given input displacement of the element. Furthermore, this work aims to determine the standard deviation of the flexoelectric coefficient with an initial displacement $\delta$. This paper will be beneficial to obtain the flexoelectric coefficient of the bending mode in a more accurate way. Consequently, it will be able to estimate the inputs with an accurate coefficient as a sensor.

Barium strontium titanate $\left(\mathrm{Ba}_{0.65} \mathrm{Sr}_{0.35} \mathrm{TiO}_{3}\right.$, here referred to as $\left.\mathrm{BST}\right)$ was used as the material for the sensing element [10]. BST is known to exhibit the highest flexoelectric coefficient [11-15] ever reported. For the experiment, a laser vibrometer, piezoelectric actuator, charge amplifier and lock-in amplifier were used. The structural stress analysis was performed using ANSYS Workbench 2019 R3(Ansys Inc, Canonsburg, PA, USA). The distribution of the strain gradient in the 3-D case was calculated using the results of stress and strain from FEA. The number of nodes and mesh type of the element was set to 3420 $(38 \times 18 \times 5)$ and hexahedral, respectively.

\section{Structural Analysis}

\subsection{Flexoelectricity and the Sensing Element}

The flexoelectric effect is defined as the linear coupling between the mechanical strain gradient and electric polarization in dielectric materials. For the bending mode, it is expressed as [16]

$$
P_{y}=\mu_{12} \frac{\partial \varepsilon_{x}}{\partial x_{y}}
$$

where $P_{y}$ is the flexoelectric polarization, $\mu_{12}$ is the transverse flexoelectric coefficient, and, $\varepsilon_{x}$ is the elastic strain in the $x$ direction. The following equation can be used to derive the charge output as

$$
q_{0}=\int_{0}^{l_{x}} P_{y} d A
$$

where $q_{0}$ is the charge output induced by the flexoelectric effect.

With the assumption of the quasi-1-D model, the beam theory can be used to obtain the following equation.

$$
q_{0}=\int_{0}^{l_{x}} \mu_{12} \frac{\partial \varepsilon_{x}}{\partial y} 2 l_{z} d x=3 \mu_{12} \delta \frac{l_{z}}{l_{x}}
$$

In Table 1, the properties of the BST material [6] used as the sensing element and dimensions of the BST unimorph are listed. The coordinate system and boundary condition used in the numerical analysis of the unimorph type sensing element with the bending mode are shown in Figure 1. The boundary conditions that are set in the FEA are that one 
surface where $x=0$ is fixed and others are free. The input displacement is applied to the center of the tip.

Table 1. Material properties of BST and dimensions of the BST unimorph.

\begin{tabular}{cc}
\hline Young's modulus $(\mathrm{GPa})$ & 153 \\
Poisson's ratio & 0.33 \\
$l_{\mathrm{x}}(\mathrm{mm})$ & 10.7 \\
$l_{\mathrm{y}}(\mathrm{mm})$ & 1 \\
$l_{\mathrm{z}}(\mathrm{mm})$ & 2.54 \\
\hline
\end{tabular}

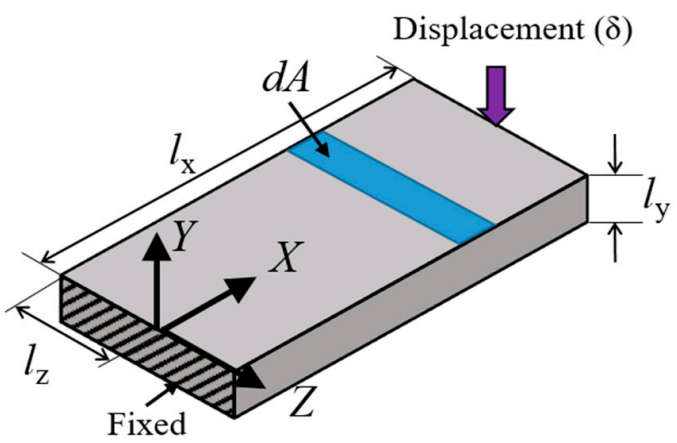

Figure 1. Configuration of the unimorph sensing element. and coordinate system used.

Figure 2 shows the arrangement of the experimental setup used to find the flexoelectric coefficient in the unimorph sensing element of the bending mode. Experiments are necessary to find the flexoelectric coefficient $\mu_{12}$ for an arbitrary concentrative force acting at the center tip of the element. The procedure to obtain the flexoelectric coefficient, which plays a key role in the estimation of the charge output for each displacement of the sensing element can be summarized as follows. Firstly, the displacement $\delta$ generated by a piezoelectric actuator is measured via a laser vibrometer. Secondly, for the case of the measured displacement, a 3-D stress and strain analysis on the given unimorph sensing element is performed; next, the values of the integrated strain gradient term are calculated using the results of the stress analysis. Thirdly, the total charge output obtained by applying the displacement $q_{0}$ is measured via a lock-in amplifier. Finally, the flexoelectric coefficients $\mu_{12}$ for the unimorph sensing element can be obtained from the results of the numerical analyses in Equation (4). Upon repeating all the steps of this process, the flexoelectric coefficients for the sensing element of the respective stress fields with various $\delta$ values can be obtained. Finally, the flexoelectric coefficient is calculated as the RMS value of the respective coefficients.

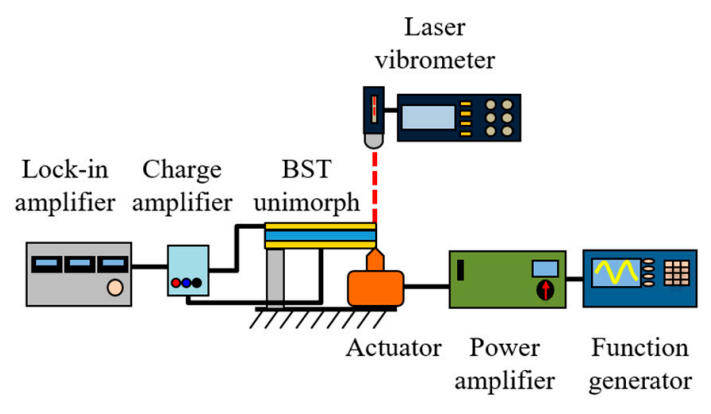

(a)

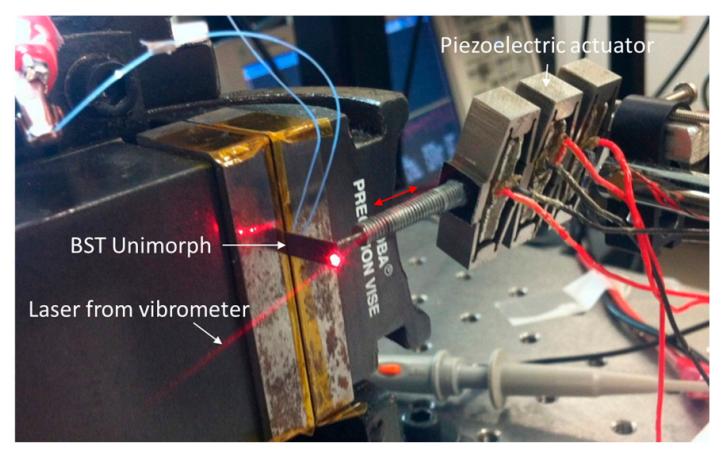

(b)

Figure 2. The experimental setups of the unimorph sensing element: (a) Whole experimental setup and (b) Actual test setup. 
As an example, for the case of $\delta=7.5 \mu \mathrm{m}$, the distributions of the stress and strain results on the upper surface of the element from FEA (ANSYS Workbench) are shown in Figure 3. It can be seen that the stress and strain are not uniformly distributed along the $z$ axis. Since the relative influence of the free surface increases with the increase of $x$, and the stress and strain decrease with the increase of $\mathrm{z}$ and $x$. It can thus be concluded that, in order to obtain a more reliable flexoelectric coefficient, the results of the 3-D stress and strain numerical analysis in the unimorph sensing element should be used.
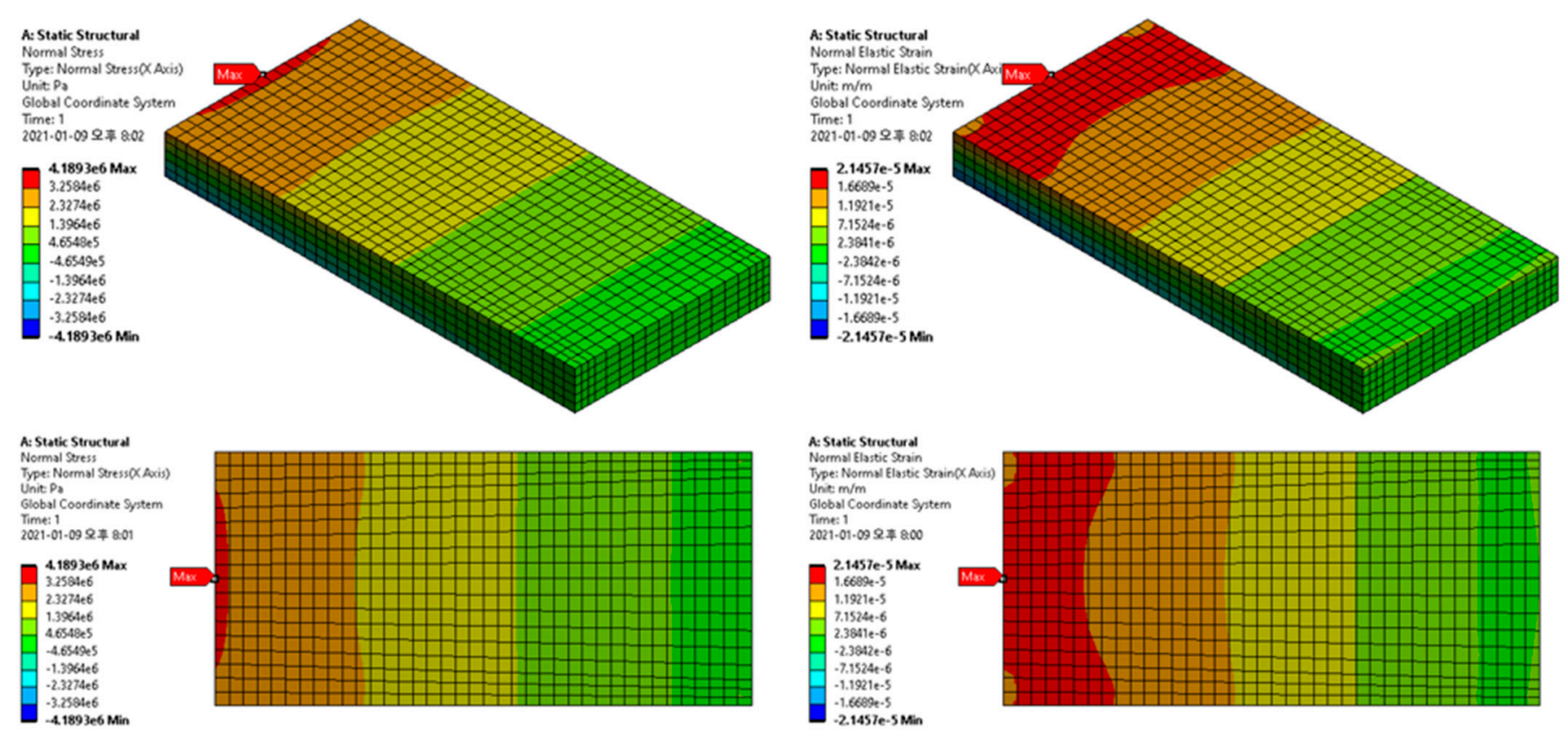

(a)

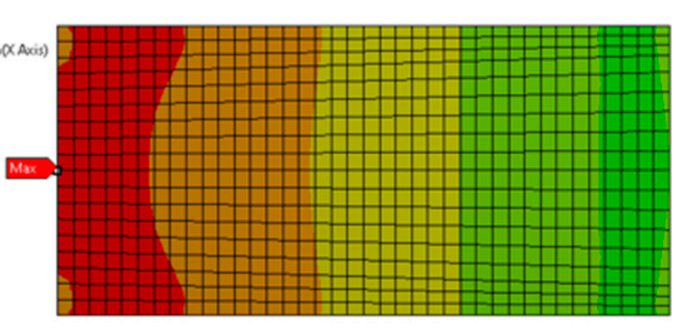

(b)

Figure 3. FEA results of the unimorph sensing element (a) stress and (b) strain.

Because ANSYS Workbench does not offer the strain gradient in the $\mathrm{Y}$ direction, the total charge due to flexoelectricity is calculated by the following steps. First, the deformation and strain due to the tip displacement of each node are obtained by FEA. Using the obtained results, the strain gradients of each element are calculated. Finally, the induced flexoelectric charge output of the unimorph is obtained by integrating all the values that are the multiplied strain gradient and flexoelectric coefficient.

\subsection{Stress}

For the case of $\delta=7.5 \mu \mathrm{m}$, the distributions of the stress $\sigma_{x}$ along the $x$ axis at various $z$ values along the upper surface of the element are shown in Figure 4 . Note that $\bar{x}, \bar{y}$ and $\bar{z}$ are normalized coordinates $\left(\bar{x}=\frac{x}{l_{x}}, \bar{y}=\frac{y}{l_{y}}\right.$ and $\left.\bar{z}=\frac{z}{l_{z}}\right)$. For simplicity, the bars on the variables will be omitted here. The average stress along the $x$ axis on the upper surface is $\frac{\sum_{k}^{n} \sigma_{x, k}}{n}$, where $n$ is the maximum number in the nodal point of $z$ at an arbitrary $x$. The 1-D result shows the stress obtained under the assumption of the quasi 1-D stress field in the sensing element. As can be seen from the figure, regardless of the $z$ value, the stress $\sigma_{x}$ decreases with the increase of $x$. Moreover, for the same $x$ value, due to the influence of the distance from the acting point of the concentrative force, the stress along the $x$ direction decreases with the increase of $z$. 


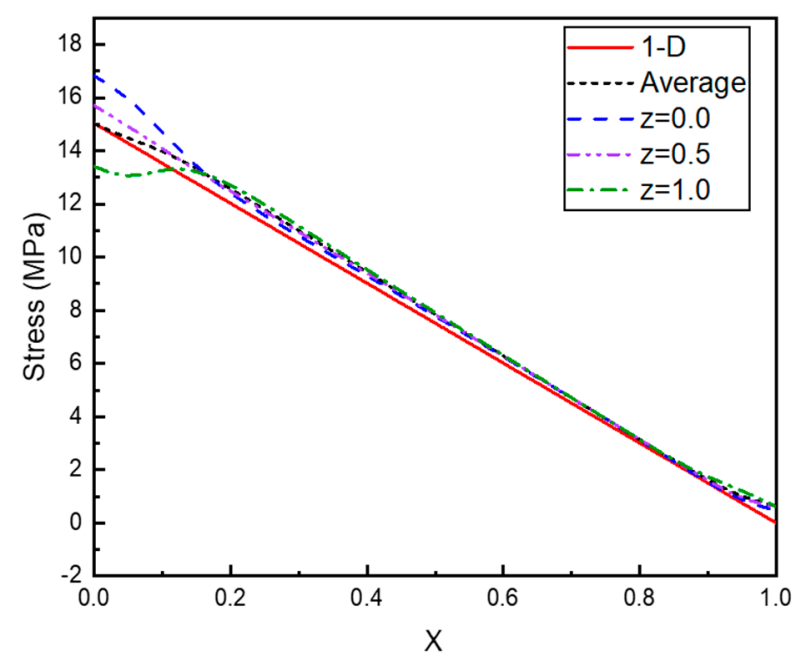

Figure 4. Distributions of stress along the $x$ axis at the upper surface of the sensing element for $\delta=7.5 \mu \mathrm{m}$.

On the other hand, the trends for both the 1-D and average 3-D stress fields in the sensing element are quite similar. However, for $x \leq 0.1$ the stress distribution obtained under the assumption of the quasi-1-D stress field is quite different from the respective stress for $z$ obtained via 3-D numerical analysis. Specifically, the stress at $z=0.0$ is $26 \%$ larger than that at $z=1.0$ on the upper surface. It is thus possible to infer that the charge outputs for the quasi-1-D and actual-3-D stress fields must be in disagreement.

\subsection{Strain}

Figure 5 shows the distributions of the strain $\varepsilon_{x}$ along the $x$ axis on the upper surface of the element under the same conditions of Figure 4. Regardless of the $z$ value, the strain $\varepsilon_{x}$ increases with increasing $x$ at first, reaches a maximum, and then decreases with a further increase of $x$. In agreement with the stress distributions in Figure 4, the maximum strain $\varepsilon_{x}$ increases with decreasing $z$. Due to the large differences in strain obtained under the assumptions of the quasi-1-D and actual-3-D stress analyses, it can be reconfirmed that, for the sake of accuracy, the 3-D numerical stress analysis should be used to determine the flexoelectricity coefficient.

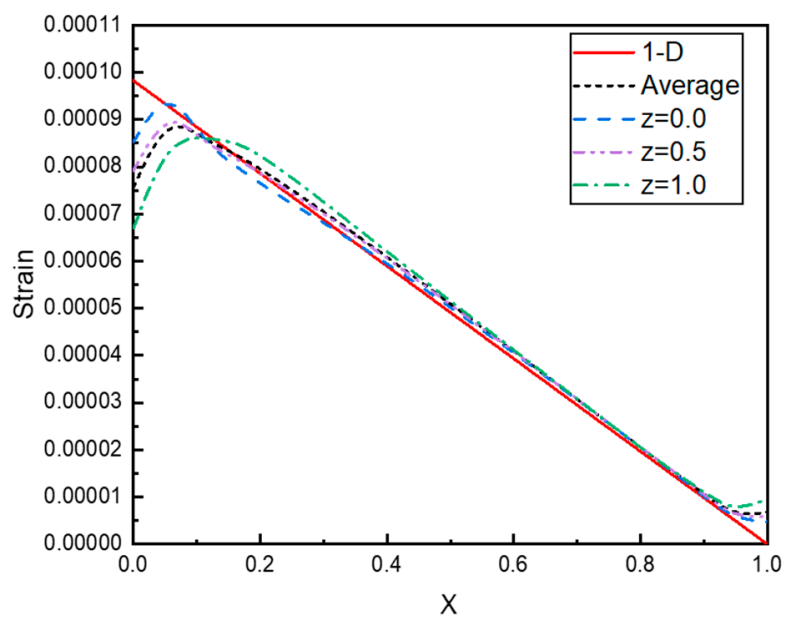

Figure 5. Strain distributions along the $x$ axis on the upper surface for $\delta=7.5 \mu \mathrm{m}$.

\subsection{Strain Gradient}

For $x=0.1$, due to the influence of the distance from the acting point of the concentrative force, the strain gradient increases with decreasing $z$ as shown in Figure 6. As an 
example, the strain gradient for $z=0.0$ and $x=0.25$ is $16 \%$ larger than that for $z=1.0$. It can thus be concluded that the effect of the deformation of the element should be taken into account for the exact calculation of the charge output.

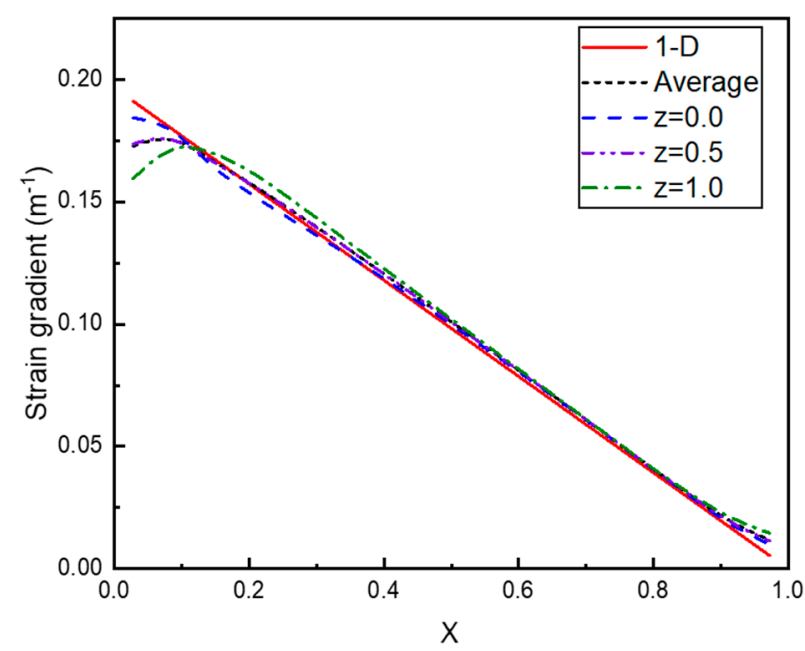

Figure 6. Strain gradient distributions along the $x$ axis on the upper surface of the element for $\delta=7.5 \mu \mathrm{m}$.

Figure 7 shows the estimated flexoelectric coefficients using both methods, quasi 1-D and 3-D stress fields. Table 2 shows the experimental results of the charge output for given displacements. In addition, with these experimental results, the coefficients estimated by two methods are listed. As can be seen from the figure and table, for the same experimental charge output (which corresponds to $\delta$ ), the average flexoelectric coefficient for the 3-D case is $1.8 \%$ smaller than that of the 1-D case. On the other hand, the standard deviations in the flexoelectric coefficients can be seen. These may be caused by measurement errors, however, from the fact that the standard deviation of the 3-D case is smaller than that of the 1-D case, it can be deduced that the proposed method for estimating the flexoelectric coefficient is more accurate.

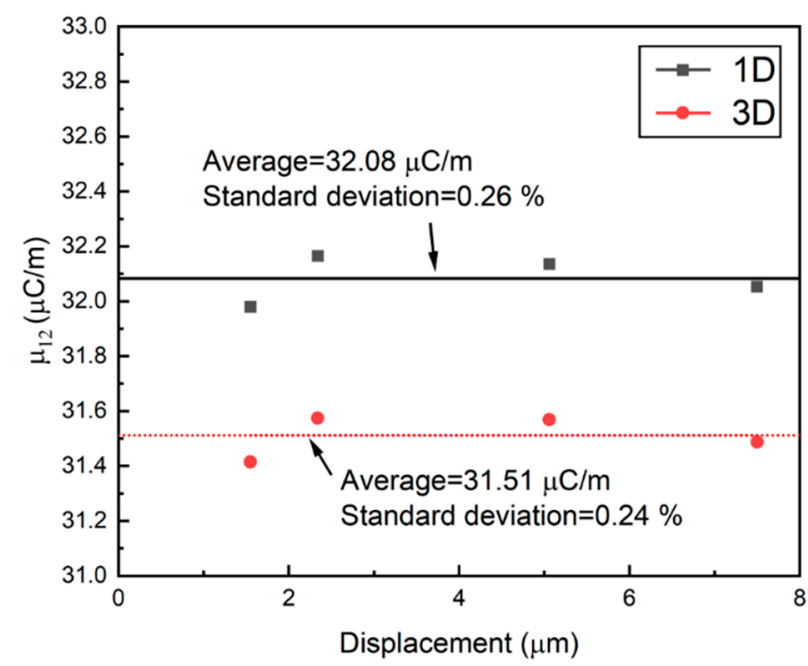

Figure 7. Comparison of flexoelectric coefficients between both approaching cases of 1-D and 3-D stress fields. 
Table 2. Experimental results of the flexoelectric charge output for various input displacements.

\begin{tabular}{cccc}
\hline Displacement $(\boldsymbol{\mu m})$ & Charge Output $(\mathbf{p C})$ & $\boldsymbol{\mu}_{\mathbf{1 2}} \mathbf{1 - D}(\boldsymbol{\mu C} / \mathbf{m})$ & $\boldsymbol{\mu}_{\mathbf{1 2}}$ 3-D $(\boldsymbol{\mu C} / \mathbf{m})$ \\
\hline 7.50 & 171.2 & 32.05 & 31.49 \\
5.06 & 115.8 & 32.14 & 31.57 \\
2.34 & 53.6 & 32.16 & 31.57 \\
1.55 & 35.3 & 31.98 & 31.41 \\
\hline \multicolumn{4}{c}{ Average } \\
\hline
\end{tabular}

\section{Discussion}

A new method was proposed to estimate more accurately the flexoelectric coefficient of the unimorph typed sensing elements. This method relies on finite element analysis (FEA) and numerical calculation. Compared to the previously used method, which is based on the calculation under the assumption of 1-D stress/strain field, the present method offers more accurate flexoelectric coefficient estimation. The estimated flexoelectric coefficient using quasi 1-D and 3-D stress fields calculated from the experimental results are compared. The average flexoelectric coefficient of the $3-\mathrm{D}$ case is $1.8 \%$ smaller than that of the 1-D case. It can be deduced that this discrepancy comes from the fact that Euler's beam theory cannot reflect the real stress field. In addition to that, despite the measurement error, less standard deviation $(0.24 \%)$ of the coefficient represents the fact that the present method offers more accuracy in the estimation of the flexoelectric coefficient. From these findings, it can be concluded that to obtain a more reliable flexoelectric coefficient, the results of the 3-D numerical stress analysis should be used.

Author Contributions: Conceptualization, methodology, validation and experiments, S.R.K., and review and editing, Y.R. All authors have read and agreed to the published version of the manuscript.

Funding: This work was supported by Basic Science Research Program through the National Research Foundation of Korea (NRF) funded by the Ministry of Science, ICT \& Future Planning (NRF-2017R1A2B4009049).

Institutional Review Board Statement: Not applicable.

Informed Consent Statement: Not applicable.

Conflicts of Interest: The authors declare no conflict of interest.

\section{References}

1. Kogan, S.M. Piezoelectric effect during inhomogeneous deformation and acoustic scattering of carriers in crystals. Sov. Phys.-Solid State 1964, 5, 2069-2070.

2. Huang, W.; Kim, K.; Zhang, S.; Yuan, F.-G.; Jiang, X. Scaling effect of flexoelectric (Ba,Sr) $\mathrm{TiO}_{3}$ microcantilevers. Phys. Status Solidi (RRL) Rapid Res. Lett. 2011, 5, 350-352. [CrossRef]

3. Eremeyev, V.A.; Ganghoffer, J.-F.; Konopińska-Zmysłowska, V.; Uglov, N.S. Flexoelectricity and apparent piezoelectricity of a pantographic micro-bar. Int. J. Eng. Sci. 2020, 149, 103213. [CrossRef]

4. Jiang, X.; Huang, W.; Zhang, S. Flexoelectric nano-generator: Materials, structures and devices. Nano Energy 2013, 2, $1079-1092$. [CrossRef]

5. Kwon, S.R.; Huang, W.B.; Zhang, S.; Yuan, F.G.; Jiang, X. Study on a flexoelectric microphone using barium strontium titanate. J. Micromech. Microeng. 2016, 26, 045001. [CrossRef]

6. Huang, W.; Kwon, S.R.; Yuan, F.G.; Zhang, S.; Jiang, X. A flexoelectric micro-accelerometer. In Proceedings of the ASME 2012 International Mechanical Engineering Congress and Exposition, Houston, TX, USA, 9-12 November 2012; pp. 597-603.

7. Huang, W.; Yan, X.; Kwon, S.R.; Zhang, S.; Yuan, F.-G.; Jiang, X. Flexoelectric strain gradient detection using $\mathrm{Ba}_{0.64} \mathrm{Sr}_{0.36} \mathrm{TiO}_{3}$ for sensing. Appl. Phys. Lett. 2012, 101, 252903. [CrossRef]

8. Kwon, S.R.; Huang, W.B.; Zhang, S.; Yuan, F.G.; Jiang, X.N. Flexoelectric sensing using a multilayered barium strontium titanate structure. Smart Mater. Struct. 2013, 22, 115017. [CrossRef]

9. Kwon, S.R. A Study on the Flexoelectric Coefficient in the Frustum Pyramid Sensing Element with the Rigid Laminates at the Upper and Bottom Surfaces. Trans. Korean Soc. Mech. Eng. A 2020, 44, 1-6. [CrossRef]

10. Ma, W.; Cross, L.E. Flexoelectric polarization of barium strontium titanate in the paraelectric state. Appl. Phys. Lett. 2002, 81, 3440-3442. [CrossRef] 
11. Chu, B.; Zhu, W.; Li, N.; Cross, L.E. Flexure mode flexoelectric piezoelectric composites. J. Appl. Phys. 2009, 106, 104109. [CrossRef]

12. Ma, W.; Cross, L.E. Flexoelectric effect in ceramic lead zirconate titanate. Appl. Phys. Lett. 2005, 86, 072905. [CrossRef]

13. Ma, W.; Cross, L.E. Flexoelectricity of barium titanate. Appl. Phys. Lett. 2006, 88, 232902. [CrossRef]

14. Ma, W.; Cross, L.E. Observation of the flexoelectric effect in relaxor $\mathrm{Pb}\left(\mathrm{Mg}_{1 / 3} \mathrm{Nb}_{2 / 3}\right) \mathrm{O}_{3}$ ceramics. Appl. Phys. Lett. 2001, 78, 2920-2921. [CrossRef]

15. Zhu, W.; Fu, J.Y.; Li, N.; Cross, L.E. Piezoelectric composite based on the enhanced flexoelectric effects. Appl. Phys. Lett. 2006, 89, 192904. [CrossRef]

16. Kwon, S.R. Study on the flexoelectric characteristics in the sensing element of a duplex frustum pyramid. J. Mech. Sci. Technol. 2018, 32, 5839-5843. [CrossRef] 